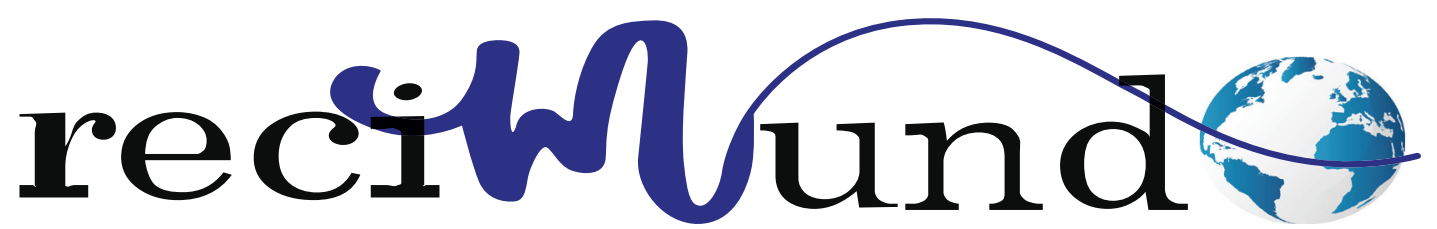

Revista Científica Mundo de la Investigación y el Conocimiento

DOI: 10.26820/recimundo/4.(4).octubre.2020.152-160

URL: http://recimundo.com/index.php/es/article/view/895

EDITORIAL: Saberes del Conocimiento

REVISTA: RECIMUNDO

ISSN: 2588-073X

TIPO DE INVESTIGACIÓN: Artículo de Revisión

CÓdIGO UNESCO: 3205 Medicina Interna; 3205.03 Gastroenterología "secHGACETCIA. LTDA.
PAGINAS: 152-160

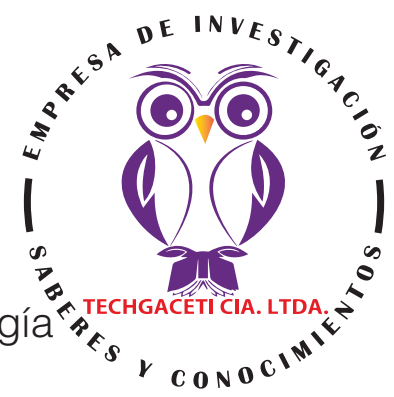

\title{
Calprotectina fecal como marcador para enfermedad inflamatoria intestinal
}

Fecal calprotectin as a marker for inflammatory bowel disease

Calprotectina fecal como marcador de doença inflamatória intestinal

Diego Rafael Mosquera Hallo; Javier Rosero Caiza2; Luis Fernando Samaniego Pineda³; Esteban Montoya Gil4

RECIBIDO: 10/07/2020 ACEPTADO: 26/08/2020 PUBLICADO: 30/10/2020

1. Médico General graduado en la Universidad Central del Ecuador; Investigador Independiente; Quito, Ecuador; dyego. mh@hotmail.com; iD https://orcid.org/0000-0003-4148-5238

2. Médico Cirujano de la Pontificia Universidad Católica del Ecuador; Investigador Independiente; Quito, Ecuador; javier. rosero94@gmail.com; (D) https://orcid.org/0000-0002-3635-6653

3. Licenciado en Laboratorio Clínico e Histotecnológico; Médico; Servicios Médicos; Institución CMED; Conocoto, Ecuador; luisfersamaniego@hotmail.com; iD https://orcid.org/0000-0002-6971-3530

4. Médico General; Hospital Baca Ortiz; Quito, Ecuador; esmongi@hotmail.com; (iD https://orcid.org/0000-0002-5861-553X

CORRESPONDENCIA

Diego Rafael Mosquera Hallo

dyego.mh@hotmail.com

Quito, Ecuador

(C) RECIMUNDO; Editorial Saberes del Conocimiento, 2020 


\section{RESUMEN}

La enfermedad inflamatoria intestinal (EII) y el síndrome del intestino irritable comparten muchos síntomas. Si bien el síndrome del intestino irritable es un trastorno funcional del intestino para el que no se dispone de un tratamiento específico, la gama de terapias eficaces para la Ell está evolucionando rápidamente. Por lo tanto, es esencial un diagnóstico preciso de la Ell. La evaluación clínica, junto con diversas modalidades de imagen y endoscopia, ha sido el pilar del diagnóstico durante muchos años. En la última década han aparecido biomarcadores fecales de inflamación gastrointestinal, de los cuales la calprotectina, una proteína citosólica de los neutrófilos, ha sido la más estudiada. La enfermedad de Crohn y la colitis ulcerosa son enfermedades crónicas remitentes y reincidente, y es importante la evaluación objetiva de la actividad de la enfermedad y la respuesta al tratamiento. Esta revisión se centra en el uso de mediciones de calprotectina fecal en el diagnóstico y seguimiento de pacientes con Ell con el fin de abordar el tema y brindar información de interés para futuras investigaciones.

Palabras clave: Calprotectina, enfermedad de Crohn, colitis ulcerosa, enfermedad inflamatoria intestinal, inflamación.

\section{ABSTRACT}

Inflammatory bowel disease (IBD) and irritable bowel syndrome share many symptoms. Although irritable bowel syndrome is a functional bowel disorder for which no specific treatment is available, the range of effective therapies for IBD is rapidly evolving. Therefore, an accurate diagnosis of IBD is essential. Clinical evaluation, along with various imaging modalities and endoscopy, has been the mainstay of diagnosis for many years. In the last decade, fecal biomarkers of gastrointestinal inflammation have appeared, of which calprotectin, a cytosolic protein of neutrophils, has been the most studied. Crohn's disease and ulcerative colitis are relapsing and remitting chronic diseases, and objective assessment of disease activity and response to treatment is important. This review focuses on the use of fecal calprotectin measurements in the diagnosis and follow-up of patients with IBD in order to address the issue and provide information of interest for future research.

Keywords: Calprotectin, Crohn's disease, ulcerative colitis, inflammatory bowel disease, inflammation.

\section{RESUMO}

A doença inflamatória intestinal (DII) e a síndrome do intestino irritável compartilham muitos sintomas. Embora a síndrome do intestino irritável seja um distúrbio funcional do intestino para o qual nenhum tratamento específico está disponível, a gama de terapias eficazes para a DII está evoluindo rapidamente. Portanto, um diagnóstico preciso de IBD é essencial. A avaliação clínica, junto com várias modalidades de imagem e endoscopia, tem sido a base do diagnóstico por muitos anos. Na última década, surgiram biomarcadores fecais de inflamação gastrointestinal, dos quais a calprotectina, uma proteína citosólica dos neutrófilos, tem sido o mais estudado. A doença de Crohn e a colite ulcerosa são doenças crônicas recorrentes e remitentes, e a avaliação objetiva da atividade da doença e da resposta ao tratamento é importante. Esta revisão enfoca o uso de medidas de calprotectina fecal no diagnóstico e acompanhamento de pacientes com DII, a fim de abordar o problema e fornecer informações de interesse para pesquisas futuras.

Palavras-chave: Calprotectina, doença de Crohn, colite ulcerosa, doença inflamatória do intestino, inflamação. 


\section{Introducción}

Los síntomas del intestino inferior, que incluyen dolor abdominal crónico o malestar con diarrea o estreñimiento, son características de presentación comunes tanto en entornos de atención primaria como secundaria. Estos síntomas pueden ser causados por una serie de afecciones diferentes, incluida la enfermedad inflamatoria intestinal (EII), de las cuales la colitis ulcerosa (CU) y la enfermedad de Crohn (EC) son las más comunes, al igual que el síndrome del intestino irritable (SII).

El SII es un trastorno intestinal funcional para el que no existe una causa identificable o una patología distintiva, y el tratamiento es sintomático. Aunque puede ser lo suficientemente molesto como para interferir con las actividades diarias normales, rara vez se asocia con una morbilidad grave. Se ha estimado que la prevalencia del SII se encuentra "entre el $10 \%$ y el $20 \%$ de la población adulta. La edad más común es entre los 20 y los 30 años, aunque se observa cada vez más en edades avanzadas y es dos veces más común en mujeres que en hombres". (National Institute for Health and Clinical Excellence, 2013)

La Colitis Ulcerosa (CU) y la enfermedad de Crohn (EC) son las dos formas más comunes de enfermedad inflamatoria intestinal (EII). "La prevalencia de CU es de aproximadamente 100 a 200 por 100.000 personas y la prevalencia de EC es de aproximadamente 50 a 100 por 100.000 personas sin diferencia significativa entre sexos" (National Institute for Health and Clinical Excellence, 2013). La edad típica de presentación es entre los 15 y los 30 años, pero hasta en un $20 \%$ se presenta durante la niñez. Sin embargo.

Tanto la CU como la EC son afecciones remitentes con un curso variable de progresión. El pronóstico de los pacientes con EC es peor que el de la CU, aunque se estima que el $10 \%$ de los pacientes con CU requerirán una colectomía en los 10 años posteriores al diagnóstico. Debido a la importancia de hacer un diagnóstico de Ell versus IBS, ha habido un gran interés en desarrollar biomarcadores que puedan ayudar al diagnóstico. (Sambuelli \& Negreira, 2019)

La vía de diagnóstico convencional incluye "análisis de sangre inicial, con hemograma completo para excluir anemia, marcadores de inflamación como la velocidad de sedimentación globular (VSG) y la proteína C reactiva (PCR), además de pruebas serológicas para la enfermedad celíaca (típicamente anticuerpos transglutaminasa tisular" (Sambuelli \& Negreira, 2019). La VSG y la PCR, aunque son específicas para la inflamación, no pueden localizarla en el intestino. Estos análisis de sangre iniciales se utilizan para ayudar a decidir qué pacientes deben proceder a los estudios de imágenes y / o endoscopia.

\section{Metodología}

Para el desarrollo de este proceso investigativo, se plantea como metodología la encaminada hacia una orientación científica particular que se encuentra determinada por la necesidad de indagar en forma precisa y coherente una situación, en tal sentido Davila, (2015) define la metodología "como aquellos pasos anteriores que son seleccionados por el investigador para lograr resultados favorables que le ayuden a plantear nuevas ideas" (p.66)

Lo citado por el autor, lleva a entender que el desarrollo de la acción investigativa busca simplemente coordinar acciones enmarcadas en una revisión bibliográfica con el fin de complementar ideas previas relacionadas Calprotectina fecal como marcador para enfermedad inflamatoria intestinal. a través de una revisión de literatura, para así finalmente elaborar un cuerpo de consideraciones generales que ayuden a ampliar el interés propuesto. 


\section{Tipo de Investigación}

Dentro de toda práctica investigativa, se precisan acciones de carácter metodológico mediante las cuales se logra conocer y proyectar los eventos posibles que la determinan. En este sentido, la presente investigación corresponde al tipo documental, definido por Castro (2016), "se ocupa del estudio de problemas planteados a nivel teórico, la información requerida para abordarlos se encuentra básicamente en materiales impresos, audiovisuales y / o electrónicos". (p.41).

En consideración a esta definición, la orientación metodológica incluye la oportunidad de cumplir con una serie de actividades inherentes a la revisión y lectura de diversos documentos, donde se encuentran ideas explicitas relacionadas con los tópicos encargados de identificar una característica inmersa en el estudio. Por lo tanto, se realizaron continuas interpretaciones con el claro propósito de revisar aquellas apreciaciones propuestas por diferentes investigadores en relación al tema de interés, para luego dar la respectiva argumentación a los planteamientos, en función a las necesidades encontradas en la investigación, apoyados en las herramientas tecnológicas para la búsqueda de trabajos con valor científico disponibles en la web que tenían conexión con el objetivo principal de la investigación.

\section{Fuentes Documentales}

El análisis correspondiente a las características que predomina en el tema seleccionado, llevan a incluir diferentes fuentes documentales encargadas de darle el respectivo valor científico y en ese sentido cumplir con la valoración de los hechos a fin de generar nuevos criterios que sirven de referencia a otros procesos investigativos. Para Castro,(2016) las fuentes documentales incorporadas en la investigación documental o bibliográfica, "representa la suma de materiales sistemáticos que son revisados en forma rigurosa y profunda para llegar a un análisis del fenómeno" (p.41). Por lo tanto, se procedió a cumplir con la lectura previa determinada para encontrar aquellos aspectos estrechamente vinculados con el tema, con el fin de explicar mediante un desarrollo las respectivas apreciaciones generales de importancia.

\section{Técnicas para la Recolección de la Infor- mación}

La conducción de la investigación para ser realizada en función a las particularidades que determinan a los estudios documentales, tiene como fin el desarrollo de un conjunto de acciones encargadas de llevar a la selección de técnicas estrechamente vinculadas con las características del estudio. Bolívar, (2015), refiere, que es "una técnica particular para aportar ayuda a los procedimientos de selección de las ideas primarias y secundarias". (p.71).

Tal como lo expresa, Bolívar, (2015) "Las técnicas documentales proporcionan las herramientas esenciales y determinantes para responder a los objetivos formulados y llegar a resultados efectivos" (p. 58). Es decir, para responder con eficiencia a las necesidades investigativas, se introdujeron como técnica de recolección el método inductivo, que hizo posible llevar a cabo una valoración de los hechos de forma particular para llegar a la explicación desde una visión general. El autor Bolívar, (2015) tambien expresa que las técnicas de procesamiento de datos en los estudios documentales "son las encargadas de ofrecer al investigador la visión o pasos que deben cumplir durante su ejercicio, cada una de ellas debe estar en correspondencia con el nivel a emplear" (p. 123). Esto indica, que para llevar a cabo el procesamiento de los datos obtenidos una vez aplicadas las técnicas seleccionadas, tales como: fichas de resumen, textual, registros descriptivos entre otros, los mismos se deben ajustar al nivel que ha sido seleccionado. 


\section{Resultados}

La calprotectina es una pequeña proteína que se une al calcio y es miembro de la familia S100 de proteínas que se unen al zinc, siendo un heterodímero de S100A8 / A9. Fue descubierto "por primera vez en 1980 y se encontró que contribuía con el $60 \%$ del contenido proteico del citosol en neutrófilos, también se ha detectado en monocitos y macrófagos, aunque en concentraciones más bajas que en neutrófilos y puede tener propiedades antimicrobianas" (Lamonte, 2010).

En presencia de inflamación intestinal activa, los neutrófilos polimorfonucleares migran a la mucosa intestinal desde la circulación.

Cualquier alteración de la arquitectura de la mucosa debido al proceso inflamatorio da como resultado la fuga de neutrófilos y, por lo tanto, calprotectina, hacia la luz y su posterior excreción en las heces. Se ha demostrado que la concentración de calprotectina en las heces se correlaciona bien con este tipo de patología. (Gajownik, 2017)

De acuerdo con (Ballard \& Ison, 2014) "la calprotectina parece distribuirse de manera homogénea en las heces y es estable hasta por 7 días a temperatura ambiente". Las concentraciones de calprotectina fecal también pueden variar con la edad. Los bebés recién nacidos tienen concentraciones de calprotectina fecal más altas que disminuyen a valores adultos a la edad de 5 años.

\section{Métodos para la medición de calprotecti- na fecal}

Los métodos para la medición de la calprotectina fecal se basan todos en técnicas inmunoquímicas que utilizan anticuerpos policlonales o monoclonales dirigidos a varios epítopos de la molécula de calprotectina dimérica.
Se pueden dividir en las que producen un resultado cuantitativo y las que producen un resultado positivo o negativo, es decir, cualitativo. Estos últimos están diseñados principalmente para su uso en el punto de atención y el resultado se puede leer visualmente con un dispositivo de medición. Los métodos iniciales eran todos técnicas de ensayo inmunoabsorbente ligado a enzimas (ELISA), pero los ensayos comerciales ahora están ampliamente disponibles y algunos se enumeran en la Tabla 1. (Labaere D, 2014)

Los ELISA disponibles comercialmente son todos muy similares y se pueden realizar manualmente o semiautomatizados utilizando varias plataformas ELISA. Los autores Kok, Elias, \& Witteman, (2012) expresan que "una desventaja del formato ELISA es que son una forma de análisis por lotes, con un lote típico de 35 a 40 muestras. Esto tiene el efecto de restringir el tiempo de respuesta para las mediciones de calprotectina". Varios fabricantes han lanzado métodos inmunoquímicos de acceso aleatorio basados en la detección fluorescente, quimioluminiscente o inmunoturbidimétrica en los últimos años.

El rendimiento analítico de todos los ensayos cuantitativos es similar, pero los métodos de acceso aleatorio permiten una mayor flexibilidad en el tamaño de los lotes y, por lo tanto, en los tiempos de respuesta. Recientemente se ha publicado una comparación de seis métodos de calprotectina fecal varios fabricantes han lanzado métodos inmunoquímicos de acceso aleatorio basados en la detección fluorescente, quimioluminiscente o inmunoturbidimétrica en los últimos años. 
Tabla 1. Algunos ensayos disponibles comercialmente para la calprotectina fecal.

\begin{tabular}{|c|c|}
\hline Fabricante & Prueba \\
\hline \multirow[t]{4}{*}{ Bühlmann } & $\begin{array}{l}\text { EK-CAL ELISA - cuantitativo (dos } \\
\text { versiones) }\end{array}$ \\
\hline & $\begin{array}{l}\text { Quantum Blue - cuantitativo rápido } \\
\text { inmunocromatografía (dos versiones) }\end{array}$ \\
\hline & $\begin{array}{l}\text { FCAL Turbo - inmunoturbidimétrico } \\
\text { cuantitativo }\end{array}$ \\
\hline & $\begin{array}{l}\text { Calpro ELISA - cuantitativo (dos } \\
\text { versiones) }\end{array}$ \\
\hline \multirow[t]{2}{*}{ Eurospital } & ELISA de Calprest - cuantitativo \\
\hline & $\begin{array}{l}\text { Calfast - inmunocromatografía } \\
\text { cuantitativa rápida }\end{array}$ \\
\hline \multicolumn{2}{|l|}{ Immundiagn } \\
\hline ostik & ELISA - cuantitativo \\
\hline \multirow[t]{2}{*}{ Phadia } & $\begin{array}{l}\text { Immunocap Elia Calprotectin - } \\
\text { cuantitativo }\end{array}$ \\
\hline & $\begin{array}{l}\text { inmunoensayo enzimático de } \\
\text { fluorescencia }\end{array}$ \\
\hline Preventis & $\begin{array}{l}\text { Prueba inmunocromatográfica } \\
\text { semicuantitativa }\end{array}$ \\
\hline \multirow{4}{*}{ Diasorina } & Liaison XL - quimioluminiscente \\
\hline & cuantitativo \\
\hline & inmunoensayo \\
\hline & $\begin{array}{l}\text { Certest - inmunocromatografía } \\
\text { semicuantitativa }\end{array}$ \\
\hline
\end{tabular}

Fuente: Los autores

Todos los métodos para la calprotectina fecal requieren alguna forma de pretratamiento de la muestra para extraer la calprotectina de las muestras de heces en un tampón para su análisis. El método de extracción "estándar de oro" es pesar una cantidad determinada de heces en el tampón. Obviamente, esta es una técnica que requiere mucha mano de obra y varios dispositivos de extracción están disponibles comercialmente. "Se ha informado que estos tienen recuperaciones variables según la consistencia de las heces con recuperaciones más bajas de muestras de heces acuosas" (Whitehead, French, Brookes, Ford, \& Gama, 2013, pág. 53)

\section{Diferenciación de SII de EII}

Los pacientes con síndrome del intestino irritable (SII) o con enfermedad inflamato- ria intestinal (EII), generalmente tienen síntomas en común como dolor abdominal, hinchazón y diarrea. Los estudios iniciales de la calprotectina fecal se centraron en su capacidad para distinguir entre estas dos patologías en pacientes remitidos a servicios de gastroenterología desde la atención primaria.

En un estudio realizado por Tibble, Sigthorsson, \& Foster, (2002) con 602 pacientes consecutivos que asistían a una clínica de gastroenterología en el King's College Hospital (Londres, Reino Unido), se midió "la calprotectina fecal y se evaluó el síndrome del intestino irritable mediante el cuestionario ROME. La calprotectina fecal tuvo una sensibilidad del $89 \%$ y una especificidad del $79 \%$ para la detección de la enfermedad intestinal orgánica" (p. 450). La sensibilidad de un cuestionario ROME positivo para SII fue del $85 \%$ con una especificidad del $71 \%$.

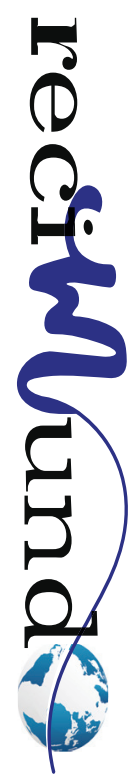


La combinación de los dos, sin embargo, tuvo un valor predictivo para SIl cercano al $100 \%$.

\section{Evaluación de la actividad de la enferme- dad y respuesta al tratamiento}

\section{Evaluación de la actividad de la enfermedad}

La calprotectina presenta una diferencia marcada entre la Ell activa e inactiva. Sin embargo la calprotectina fecal se relaciona con la actividad de la enfermedad al ser evaluada endoscópicamente. De acuerdo con Schoepfer, Beglinger, \& Strauman, (2010) "en un estudio en pacientes con Ell, la calprotectina fecal se correlacionó bien con la actividad de la enfermedad endoscópica medida mediante el índice de Rachmilewitz $(r=0,834) "$ ( $p .162)$. Un estudio similar en la CU realizado por Schoepfer, Beglinger, \& Strauman, Fecal, (2013) utilizando el índice de Baron modificado, "también se encontró que la calprotectina fecal se correlacionó mejor con la actividad de la enfermedad endoscópica que la PCR $(r=0,821$ frente a 0,556)" (p. 332).

\section{Evaluación de la respuesta al tratamiento}

El objetivo del tratamiento en la Ell es asegurar y mantener la remisión, para lograr la curación de la mucosa. "La farmacoterapia incluye esteroides, amino salicilatos, tiopurinas, metotrexato, inmunosupresores (p. Ej., Ciclosporina) y anticuerpos dirigidos contra el factor de necrosis tumoral alfa (TNF-alfa) (p. Ej., Infliximab). La evaluación de la eficacia del tratamiento se ha basado tradicionalmente en el alivio subjetivo de los síntomas, puntuaciones clínicas como el índice de actividad de la enfermedad de Crohn (CDAI) y el índice de actividad de la enfermedad de colitis ulcerosa (UCDAI) y cambios en la VSG y / o la concentración de PCR. (Alicia, Silvia, \& Anibal, 2019, pág. 34).

\section{Pronóstico}

Predicción de la recaída de la enfermedad

De acuerdo con (Vázquez, 2017) "la calprotectina fecal puede predecir la recaída de la Ell en los siguientes 12 meses en pacientes en remisión". Sin embargo, se han propuesto una variedad de límites para detectar la recaída y esto va a depender en parte de si los autores optaron por maximizar la sensibilidad o la especificidad. Cuanto menor sea el punto de corte, mayor será la sensibilidad a expensas de la especificidad y viceversa.

\section{Recurrencia posoperatoria de la Ell}

Los autores Gisbert, Bermejo, \& Perez, (2009) exponen que "el tratamiento quirúrgico de la EC con resección del íleon terminal o colectomía en la CU puede ser necesario hasta en un $20 \%$ de los pacientes durante su vida" (p. 15). Las recomendaciones actuales son que en pacientes con EC tras resección ileocolónica, la colonoscopia debe realizarse 6-12 meses después de la cirugía. Sería deseable un biomarcador no invasivo con buen poder predictivo para identificar aquellos sin recurrencia y de tal manera minimizar las molestias y el malestar del paciente.

Varios estudios han demostrado que "las concentraciones de calprotectina fecal permanecen altas después de la cirugía en aquellos sujetos que posteriormente desarrollaron recurrencia en comparación con aquellos en remisión cuyos valores a menudo caían dentro del rango de referencia”. (Gisbert, Bermejo, \& Perez, 2009). Los pacientes que se han sometido a colectomía por CU tienen un riesgo significativo de desarrollar pouchitis después de la cirugía. Hay solo unos pocos estudios sobre el valor de la calprotectina fecal en la detección de pacientes con pouchitis. 


\section{Otros marcadores fecales}

La calprotectina ha sido el marcador fecal de inflamación del tracto gastrointestinal más estudiado, pero en los últimos años han aparecido otros marcadores que pueden resultar superiores o proporcionar información complementaria. "Estas incluyen otras proteínas expresadas en el citoplasma de los neutrófilos, como lactoferrina, S100A12, elastasa polimorfonuclear y M2-PK, que es una isoforma de piruvato quinasa expresada por células que se dividen rápidamente. Estos han sido revisados recientemente". (Wright, Kamm, \& De Cruz, 2015)

\section{Conclusión}

Durante el desarrollo del proceso investigativo se observó que el uso potencial de la medición de calprotectina fecal se realiza a través de un diagnostico preciso para identificar la sensibilidad y especificidad adecuada de la calprotectina fecal con el objetivo de que se logre determinar a los pacientes con mayor probabilidad de tener enfermedad orgánica intestinal a través del manejo eficaz del examen de colonoscopia.

Lo anteriormente mencionado se logra por medio de una evaluación de la actividad de la enfermedad y su respuesta al tratamiento con la finalidad de lograr la curación de la mucosa que se puede identificar de forma no invasiva mediante la normalización de la calprotectina fecal. Sin embargo, una calprotectina fecal en aumento puede predecir una recaída clínica inminente de la Ell, lo que permite el inicio rápido del tratamiento. No obstante, se ha descubierto que varias enfermedades intestinales tienen cierto grado de inflamación mediante el uso de mediciones de calprotectina fecal, incluso cuando no se sospechaba originalmente.

\section{Bibliografía}

Alicia, S., Silvia, N., \& Anibal, G. (2019). manejo de la enfermedad inflamatoria intestinal. revisión y algoritmos de tratamiento. Acta de Gastroenterologia.
Ballard, R., \& Ison, C. (2014). Diagnóstico de laboratorio de las infecciones de transmisión sexual, incluida la infección por el virus de la inmunodeficiencia humana. Organizacion Mundial de la Salud.

Gajownik, Ú. (2017). Evaluación de la calprotectina fecal en la detección de la actividad de la enfermedad inflamatoria intestinal del colon. En la Universidad de Murcia ( España ) : Trabajo de Grado.

Gisbert, P., Bermejo, F., \& Perez, L. (2009). Calprotectina fecal y lactoferrina para la predicción de la recaída de la enfermedad inflamatoria intestinal. Inflamm Intestinal Dis. , 1190-1198.

Kok, L., Elias, S., \& Witteman, B. (2012). Diagnostic accuracy of point-of-care fecal calprotectin and immunochemical occult blood tests for diag-nosis of organic bowel disease in primary care: the Cost-Effectiveness of a Decision Rule for Abdominal Complaints in Primary Care (CEDAR) study. Clin Chem., 58:989-998.

Labaere D, S. A. (2014). Comparison of six different calprotectin assays for the assessment of inflammatory bowel disease. United European Gastroenterol $\mathrm{J}$.

Lamonte, B. (23 de Enero de 2010). Tecnicas de Laboratorio en Inmonologia Clinica. Recuperado el 09 de Octubre de 2020, de www.medic.ula.ve

National Institute for Health and Clinical Excellence. (2013). Faecal calprotectin diagnostic tests for inflammatory diseases of the bowel. London UK: NICE diagnosticguideline 11

Sambuelli, A. M., \& Negreira, S. (2019). manejo de la enfermedad inflamatoria intestinal. revisión y algoritmos de tratamiento. Acta Gastroenterologia Latinoamericana.

Schoepfer, A., Beglinger, C., \& Strauman, A. (2010). Fecal calprotectin corre-lates more closely with the Simple Endoscopic Score for Crohn's disease (SES-CD) than CRP, leukocytes and the CDAI. Am $\mathrm{J} \neg$ Gastroenterol., 105:162-169.

Schoepfer, A., Beglinger, C., \& Strauman, A. (2013). Fecal calprotectin more accurately reflects endoscopic activity of ulcerative colitis than the Lichtiger Index, C-reactive protein, platelets, hemoglobin and blood leukocytes. Inflamm Bowel Dis, 19:3323.

Tibble, J., Sigthorsson, G., \& Foster, R. (2002). . Use of surrogate markers of inflammation and Rome criteria to distin-guish organic from nonorganic intestinal disease. . Gastroenterol., 123:450-460.

Vázquez, M. (17 de 11 de 2017). Utilidad de la cal-

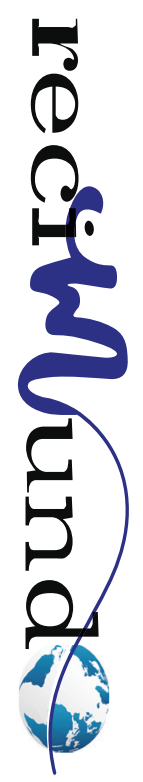


protectina fecal en la enfermedad inflamatoria intestinal. Recuperado el 11 de 10 de 2020, de Sociedad Andaluza de Patologia Digestiva: https:// www.sapd.es/revista/2017/40/2/02

Whitehead, S., French, J., Brookes, M., Ford, C., \& Gama, R. (2013). Between-assay variability of faecal calprotectin enzyme-linked immunosorbent assay kits. Ann Clin Biochem.
Wright, E., Kamm, M., \& De Cruz, P. (2015). Measurement of fecal improves monitoring and detection of recurrence of Crohn's disease after surgery. Gastroenterol., 938-947.

\section{CitAR ESTE ARTICULO:}

Mosquera Hallo, D. R., Rosero Caiza, J., Samaniego Pineda, L. F., \& Montoya Gil, E. (2020). Calprotectina fecal como marcador para enfermedad inflamatoria intestinal. RECIMUNDO, 4(4), 152-160. https://doi.org/10.26820/recimundo/4.(4).octubre.2020.152-160 